\title{
Manipulating the momentum state of a condensate by sequences of standing wave pulses
}

\author{
Wei Xiong, Xuguang Yue, Zhongkai Wang, Xiaoji Zhou ** and Xuzong Chen† \\ School of Electronics Engineering \& Computer Science, Peking University, Beijing 100871, China
}

(Dated: October 28, 2018)

\begin{abstract}
We analyze the effects of sequences of standing wave pulses on a Bose-Einstein condensate (BEC). Experimental observations are in good agreement with a numerical simulation based on the band structure theory in the optical lattice. We also demonstrate that a coherent control method based on such sequences of pulses is very efficient for experimentally designing specific momentum states.
\end{abstract}

PACS numbers: $67.85 . \mathrm{Hj}, 67.85 . J \mathrm{k}, 03.75 . \mathrm{Kk}$

\section{INTRODUCTION}

Atomic interferometry is very useful in fundamental studies of coherence, decoherence and phase shifts and for practical precision measurements, with the example of gravimeters, gyroscopes, and gradiometers [1]. BoseEinstein Condensate (BEC) based atomic interferometry provides high contrasts, long integration times and the possible use of small devices [2, 3]. In such atomic interferometry, coherent momentum manipulation is very effective for splitting and recombining the condensate [46], hence realizing the interference. In some precision measurements, the accumulated phase is positively correlated to the atomic velocity, so that the larger the atomic momentum is, the more precise the measurements can be if the measuring time is unchanged.

The diffraction of atoms from standing wave light, which is usually divided into three regimes as Bragg, Raman-Nath and channeling regimes [7] by the interaction intensity and duration, is one of the common methods to coherently manipulate the momentum states of a condensate 8 11]. One-pulse Bragg or Raman-Nath scattering can be applied for momentum states' preparation, but those techniques are then limited by constraints on the pulse properties [7]. A momentum manipulation method by multi-pulse standing waves is proposed in 12], where the momentum states can be designed, but not observed yet, and the pulses are still restricted in the Raman-Nath regime.

In this paper, we apply a method for flexible manipulation of the atomic momentum states, where the standing wave pulses are less limited in pulse intensities and durations. The atomic diffraction from one, two, three and four standing wave pulses are demonstrated in our experiments and systematically analyzed by the band structure theory of one-dimension optical lattice. With this method, we are able to design and realize several specific momentum states, which may be applied in atomic in-

\footnotetext{
*Electronic address: xjzhou@pku.edu.cn

${ }^{\dagger}$ Electronic address: xuzongchen@pku.edu.cn
}

terferometry. In principle, this method could be used for designing a wide range of possible target states.

This paper is organized as follows. In Section II, a theory to interpret the scattering process by a sequence of pulses is presented, where the standing wave is treated as a one-dimensional optical lattice. We derive a concise expression for calculating the probability of each momentum state at the end of the process. In Section III, the experiments of one, two, three and four pulses' scattering are demonstrated and compared to theoretical simulations. It is found that a correction due to momentum dispersion may be introduced into the theory for a better agreement with the experimental results. Section IV presents the experimental realization of several useful momentum states by coherent control. Section V contains the discussions and the conclusions.

\section{THEORETICAL MODEL}

We first consider a non-interacting condensate being diffracted by a sequence of square shaped standing wave pulses with the successive durations $\tau_{i}(i=1,2, \ldots, s+1)$, separated by the intervals $\tau_{f i}(i=1,2, \ldots, s)$. The standing wave consists of a pair of laser beams far-detuned enough to suppress the spontaneous emission.

The periodic potential (one-dimension optical lattice) [13, 14] introduced by the ac Stark shift can be described as $V(x)=U_{0} \cos ^{2}\left(k_{L} x\right)$, with the trap depth $U_{0}$ and the laser's wave vector $k_{L}=2 \pi / \lambda_{L}\left(\lambda_{L}\right.$ is the wavelength of the laser). The lattice leads to a band structure of the energy spectra, of which the eigenvalues of the energy $E_{n, q}$ and eigenvectors $|n, q\rangle$ (Bloch states) are labeled by the quasi-momentum $q$ and the band index $n$, and they satisfy the equation:

$$
\hat{H}|n, q\rangle=E_{n, q}|n, q\rangle
$$

where the Hamiltonian $\hat{H}=\hat{p}^{2} / 2 M+U_{0} \cos ^{2}\left(k_{L} x\right)$, with the atomic momentum $\hat{p}$ and the atomic mass $M$. The Bloch states form a quasi-momentum space. In the lattice, the spatial periodicity of the wave function results in separated peaks in momentum space, corresponding to the reciprocal lattice vector $2 k_{L}$. 
When a condensate with an initial momentum $p_{m_{0}}=$ $\hbar\left(q+2 m_{0} k_{L}\right)$ ( $\hbar$ is the Plank constant, $-1 \leq q \leq 1$, $\left.m_{0}=\ldots,-1,0,1, \ldots\right)$ is abruptly loaded into a lattice, the wave packet can be described as a superposition of the Bloch states:

$$
|\Psi(t=0)\rangle=\sum_{n=0}^{\infty}|n, q\rangle\left\langle n, q \mid p_{m_{0}}\right\rangle
$$

where $\left\langle n, q \mid p_{m_{0}}\right\rangle=c_{n, q}\left(m_{0}\right)$. The $n^{t h}$ Bloch state evolves independently as $e^{-i E_{n, q} t / \hbar}$, and the total wave function evolves as

$$
|\Psi(t)\rangle=\sum_{n=0}^{\infty} c_{n, q}\left(m_{0}\right) e^{-i E_{n, q} t / \hbar}|n, q\rangle
$$

While the incident light is switched off after the duration $\tau_{1}$, the wave function is projected back to the momentum space from the quasi-momentum space. The coefficient $b\left(m_{0}, m, \tau_{1}\right)$ of each $\left|p_{m}\right\rangle$ state $(m=\ldots,-1,0,1, \ldots)$ can be acquainted as:

$$
b\left(m_{0}, m, \tau_{1}\right)=\sum_{n=0}^{\infty} c_{n, q}\left(m_{0}\right) c_{n, q}(m) e^{-i E_{n, q} \tau_{1} / \hbar}
$$

For a zero initial momentum of the condensate, the subscript $q$ can be omitted for simplification and $m_{0}=0$. For one pulse scattering, the population of the $\left|p_{m}\right\rangle$ state is $P_{m}^{(1)}=\left|b\left(0, m, \tau_{1}\right)\right|^{2}$. It can be seen that the probabilities of the momentum states after one scattering pulse depend on the lattice depth and the pulse duration. The lattice depth determines the band structure and is reflected in the terms $c_{n, q}$. The pulse duration influences the phase evolution of each Bloch state as $e^{-i E_{n, q} \tau_{1} / \hbar}$.

The multi-pulse process, which consists of a number of single pulses and intervals can be solved as follows. The wave function of the condensate after the first pulse $\tau_{1}$ can be derived from Eq. (44) as

$$
\left|\Psi\left(\tau_{1}, t\right)\right\rangle=\sum_{m} b\left(m_{0}, m, \tau_{1}\right) e^{-i E^{(m)} t / \hbar}\left|2 m \hbar k_{L}\right\rangle
$$

After the first interval $\tau_{f 1}$ and the second pulse $\tau_{2}$, the population of the $\left|p_{m}\right\rangle$ state can be achieved as

$$
P_{m}^{(2)}=\left|\sum_{m_{1}} b\left(m_{0}, m_{1}, \tau_{1}\right) e^{-i E^{\left(m_{1}\right)} \tau_{f 1} / \hbar} b\left(m_{1}, m, \tau_{2}\right)\right|^{2}
$$

As shown in Eq. (6), the population is affected by the two pulses, the first one corresponding to $b\left(m_{0}, m_{1}, \tau_{1}\right)$, and the second one corresponding to $b\left(m_{1}, m, \tau_{2}\right)$. During the interval $\tau_{f 1}$, the phase of the $\left|p_{m_{1}}\right\rangle$ state evolves along the time as $e^{-i E^{\left(m_{1}\right)} t / \hbar}$, where $E^{\left(m_{1}\right)}=\left(2 m_{1} \hbar k_{L}\right)^{2} / 2 M=$ $4 m_{1}^{2} E_{R}$ is the kinetic energy, and $E_{R}=\left(\hbar k_{L}\right)^{2} / 2 M$ is the single photon recoil energy. The interval $\tau_{f 1}$ produces a phase shift $e^{-i E^{(m)} \tau_{f 1} / \hbar}$ and contributes to the momentum distribution.
In the same way, the population of the $\left|p_{m}\right\rangle$ state after $(s+1)$ pulses can be achieved as:

$$
P_{m}^{(s+1)}=\left|\sum_{m_{1}, m_{2}, \cdots, m_{s}} \prod_{i=1}^{s+1} b\left(m_{i-1}, m_{i}, \tau_{i}\right) \prod_{i=1}^{s} e^{-i E^{\left(m_{i}\right)} \tau_{f_{i}} / \hbar}\right|^{2}
$$

with $m_{0}=0$, and $m_{s+1}=m$.

From the analysis above, the momentum distribution after a sequence of pulses' scattering is influenced by not only the lattice pulses with the term $\prod_{i=1}^{s+1} b\left(m_{i-1}, m_{i}, \tau_{i}\right)$, but also the intervals among the pulses as reflected in the term $\prod_{i=1}^{s} e^{-i E^{\left(m_{i}\right)} \tau_{f_{i}} / \hbar}$. Although the populations of the momentum states do not change during the intervals, the phase-evolution rates of the momentum states with different kinetic energies are not identical. The phase deviations between the states oscillate from 0 to $2 \pi$ with the interval, and the heterogeneously accumulated phases change the distribution of the condensate in the quasi-momentum space.

\section{EXPERIMENTS OF STANDING WAVE PULSE SEQUENCES}

We performed the experiments of a condensate in a magnetic trap (MT) (see Fig. 1(a)) being scattered by a sequence of standing wave pulses (see Fig. 1(b)). As shown in Fig. 1(c), after pre-cooling, a cigar shaped ${ }^{87} \mathrm{Rb}$ condensate of $2 \times 10^{5}$ atoms in $5^{2} S_{1 / 2}\left|F=2, M_{F}=2\right\rangle$ state was achieved by the radio frequency $(\mathrm{RF})$ cooling in the magnetic trap, of which the axial frequency is $20 \mathrm{~Hz}$ and the radial frequency is $220 \mathrm{~Hz}$ [15, 16]. A pair of counter-propagating laser beams, of which the durations were controlled by an acousto-optical modulator, and the amplitudes were adjusted by the injection current of a tapered-amplifier, were applied to the condensate along the axial direction. The linear polarized incident light at the wavelength $\lambda_{L}=852 \mathrm{~nm}$ was focused with a waist of $110 \mu \mathrm{m}$ to cover the condensate. The trap depth, which was calibrated by Kapitza-Dirac scattering experimentally, reached $120 E_{R}$, corresponding to the light power of $320 \mathrm{~mW}$. The incident light and the magnetic trap were simultaneously shut after the BEC-light interaction. After $30 \mathrm{~ms}$ free falling and ballistic expansion, the momentum distribution of the condensate was pictured by absorption imaging.

The lattice in our experiments is quite deep, so we concentrate on the short-pulse diffractions to avoid the de-coherence and heating effects of long pulses relevant for Bragg scattering. However, for more flexible momentum manipulation, our pulses are not so short as the Raman-Nath pulses [17] used in previous works.

A brief instruction to the Raman-Nath regime is given in the following for comparison. In the scattering process, the evolution during the free evolution intervals is analyzed as in previous section, while the effect of lattice with 
(a)

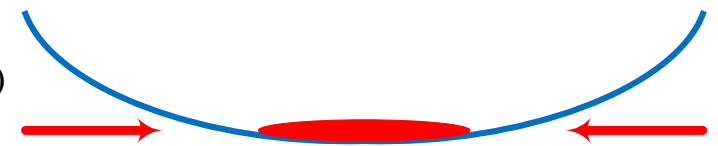

(b)

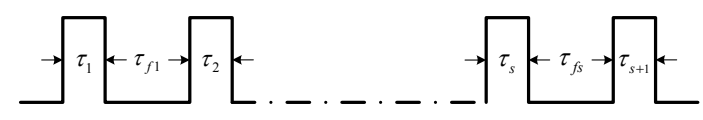

(c)

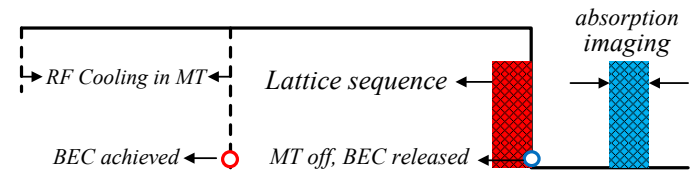

FIG. 1: (a) A pair of counter-propagating light beams are applied to a condensate in magnetic trap. (b) The scattering process consists of a sequence of standing wave pulses, which contained $s$ intervals with widths $\tau_{f i}(\mathrm{i}=1,2, \ldots s)$ and $s+1$ light pulses with durations $\tau_{i}(\mathrm{i}=1,2, \ldots s+1)$. The incident light's wavelength is $852 \mathrm{~nm}$ and its maximum intensity can reach $120 E_{R}$. (c) The procedure for the experiments is shown. The condensate is exposed to a sequence of standing wave pulses and then released from the magnetic trap. The absorption images of the condensate can be observed after the free falling.

adequately short duration $\tau$ can be analytically solved by the Schrödinger equation $i \partial|\Psi(t)\rangle / \partial t=\hat{H}|\Psi(t)\rangle$, after omitting the atomic kinetic energy term $\hat{p}^{2} / 2 M$ in the Hamiltonian. This approximation can be made while the displacement of the scattered atoms during the interaction time is much smaller than the spatial period of the standing wave. Equivalently, the standing wave duration $\tau$ and the single photon recoil frequency $\omega_{r}=\hbar k_{L}^{2} / 2 M$ have to fulfill $\tau \ll 1 / \omega_{r}$. The pulse is able to split a stationary condensate into components with symmetrical momenta $p_{n}=2 \hbar k_{L}(n=0, \pm 1, \pm 2, \ldots)$, with corresponding populations $P_{n}=J_{n}^{2}\left(U_{0} \tau / 2 \hbar\right)$, where $J_{n}(z)$ are Bessel functions of the first kind.

First we demonstrate a one-pulse scattering experiment. A condensate is exposed to a standing wave pulse with depth $100 E_{R}$ and duration varying from 0 to $30 \mu \mathrm{s}$. The relative populations of the condensates with the momenta $0 \hbar k, \pm 2 \hbar k, \pm 4 \hbar k$ and $\pm 6 \hbar k$, corresponding to Fig. 2(a), (b), (c) and (d) respectively, are measured and theoretically analyzed. In addition, the theoretical analysis with the Raman-Nath approximation, is also shown in the figure for comparison. It can be seen that within $3 \mu s$ the theoretical analysis with the Raman-Nath approximation (blue solid line) is close to the experimental results (black dots), and so is the theoretical analysis with band structure theory (red dashed line). When the pulse duration exceeds $3 \mu s$, the analysis with the Raman-Nath approximation gradually goes far away from the experimental results, while the numerical simulation with band structure theory still agrees with the experimental results along the whole time scale. As shown in Fig. 2 the prob-

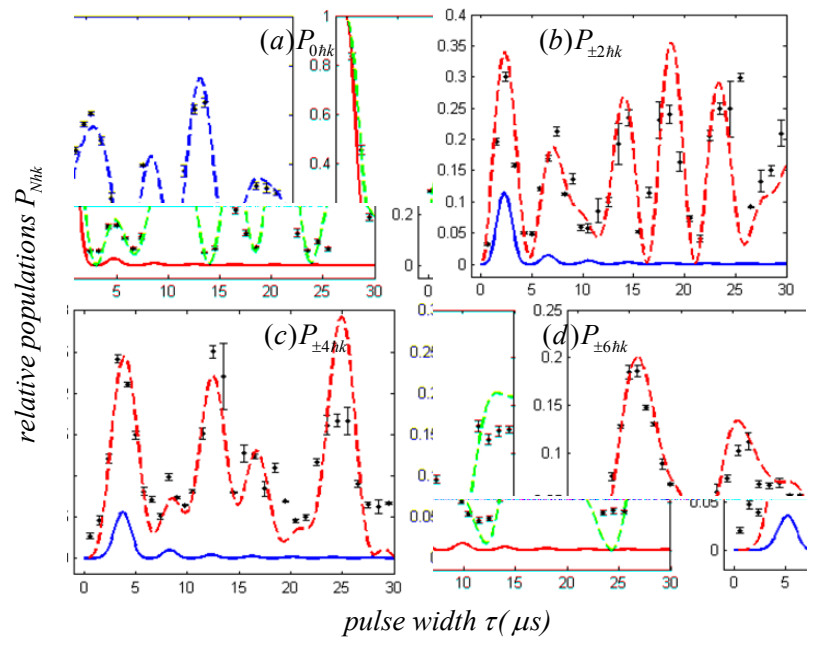

FIG. 2: Single pulse scattering of condensate: The black dots represent the experiment results. The blue solid line is the theoretical analysis with Raman-Nath approximation. The red dashed line is the numerical simulation with band structure theory. Figure (a), (b), (c) and (d) correspond to the relative populations of the condensates with the momenta $0 \hbar k$, $\pm 2 \hbar k, \pm 4 \hbar k$ and $\pm 6 \hbar k$ respectively.

ability of each momentum state oscillates with the pulse duration as described by the band structure theory. It is clear that, in the single pulse scattering process, the band structure theory works well not only for the short pulse but also for the longer pulse, because the atomic motion has been taken into account. So the atomic diffraction by a single standing wave pulse can be predicted in a wider range of pulse duration with the band structure theory.

Then we increase the number of pulses in the experiments to explore the extra factors influencing the momentum distributions. Two groups of experiments are carried out, where one consists in two two-pulse sequences and the other uses a train of three pulses or a train of four pulses. In every sequence, all the pulses are the same and all the intervals are identical to make the experiments more convenient to carry out. For further comparison between the band structure theory and the analysis in the Raman-Nath regime, every single pulse is made short enough for the Raman-Nath approximation.

Two experiments of two-pulse scattering are demonstrated in Fig. B] in which the relative populations of the stationary condensate $P_{0 \hbar k}$ versus the varied intervals $\tau$ is shown. The parameters of the scattering pulses used in different sequences are chosen to be of the same products of the lattice depth and the pulse duration, so that each pulse affects the condensate equivalently. As shown in the figure, the intervals actually affect the final momentum distribution, and the theoretical analysis with the band structure theory and Raman-Nath approximation both picture well the evolution of the atomic distributions versus the interval between the two pulses. The results of two-pulse scattering can be explained as the 

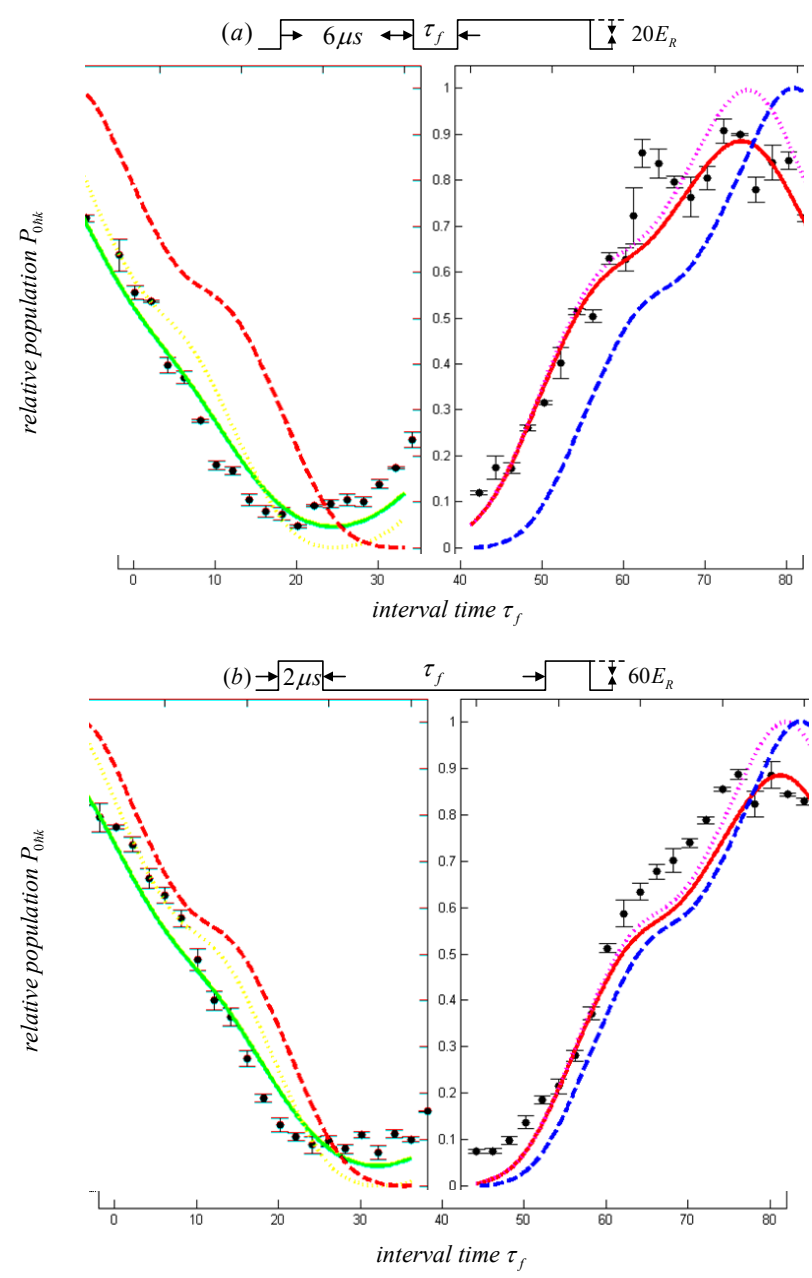

FIG. 3: Two-pulse scattering of the condensate: The relative populations of the stationary condensate $P_{0 \hbar k}$ versus the varied intervals $\tau$ is shown. The parameters of the experiments are described above each figure. The black dots are the experimental results. The blue dashed line is the analysis with Raman-Nath approximation. The magenta dotted line is the numerical simulation with band structure theory. The red solid line is a numerical simulation taking into account the momentum dispersion.

fact that since the phase shift accumulated during the interval varies harmonically from 0 to $2 \pi$, the probability of the stationary condensate oscillates between the minimum and the maximum. When the phase shift is $2 \pi$ with the interval $\pi \hbar / 2 E_{R}$ (around $80 \mu \mathrm{s}$ ), the wave function is little affected by the interval and the two pulses diffract the condensate as one combined pulse to make the probability $P_{0 \hbar k}$ the minimum. While the phase shift is $\pi$ with the interval $\pi \hbar / 4 E_{R}$ (about $40 \mu s$ ), the second pulse produces an effect opposite of the first one and diffract the non-stationary components of the condensate back to the stationary one and make the probability $P_{0 \hbar k}$ the maximum.

It also can be acquainted from Fig. 3 that the numeri- cal simulation with band structure theory is much closer to the experimental results than the analytical solution with Raman-Nath approximation. It is conjectured that the phase evolution during the scattering process makes the difference. The phase evolution in the scattering process is neglected in the Raman-Nath approximation, but not in the numerical simulation with band structure theory. Although the duration of the scattering process is short, the phase shifts in the scattering process still increase. The phase shift in the scattering process needs to be taken into account and influences the final momentum distribution. As a result, the longer the scattering pulse is, the larger the difference is. Although the maximum of the probability $P_{0 \hbar k}$ corresponds to the interval $\pi \hbar / 4 E_{R}$, the two-pulse experiments in Fig. 3 can clearly show that the longer pulse leads to the larger difference. In Fig. 3 (a), the pulse duration is $6 \mu s$, the probability $P_{0 \hbar k}$ reaches the maximum with the interval $34 \mu s$. In Fig. 3 (b), the pulse duration is $2 \mu s$, the probability $P_{0 \hbar k}$ gets to the top with the interval $38 \mu \mathrm{s}$.

Nevertheless, there is still some obvious deviation between the simulation and the experimental results. It is observed that the momentum width has been expanded after the former pulse, because of the $s$-wave scattering between the different momentum states. Consequently, this dispersion process is approximated to an initial momentum width of $\sim 0.1 \hbar k_{L}$ on average to optimize the numerical simulation. Unlike the analysis without momentum width, phase evolution is different for different initial momenta and results in a phase dispersion. The quasi modes obtained at the end of the diffraction process result from the linear superposition of final states obtained after time evolutions of the different momenta populated the initial BEC. It can be seen from Fig. 3 that the approximation is effective.

As discussed in [18], the maximum of the probability $P_{0 \hbar k}$ will never reach 1 thanks to the imperfect optical lattice. In our case, the momentum expansion is an explanation of the similar situation as shown in Fig. 3 . Since the momentum width is considered, the phase shift is populated around $\pi$ with a width, instead of a definite $\pi$, with the interval $\pi \hbar / 4 E_{R}$. In other words, there is no interval that accumulates a phase shift exactly equal to $\pi$, so with any interval, the second standing wave pulse is not able to diffract all the condensates back to the stationary part.

The experiments of one three-pulse scattering and one four-pulse scattering are demonstrated in Fig. 4, where the relative populations of the stationary condensate $P_{0 \hbar k}$ versus the varied total intervals $\sum \tau_{f i}$ is shown. In each experiment, the total interaction intensity $\sum U_{0} \tau$ is the same, with different number of the pulses. These two experiments show that even though the total interactions and intervals are the same, the different processes of phase accumulations in the two kinds of pulse sequences result in distinct momentum distributions. We directly apply the band structure theory with momentum dispersion to analyze the experiments in Fig. [4 and the 


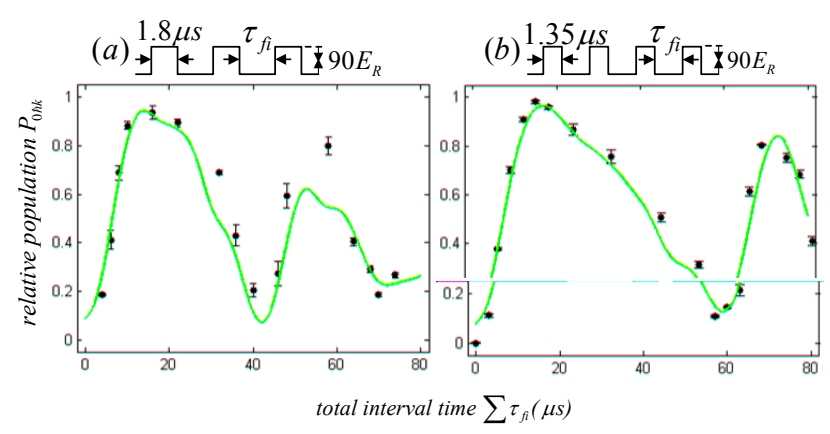

FIG. 4: Three-pulse and four-pulse scattering of the condensate: The relative populations of the stationary condensate $P_{0 \hbar k}$ versus the varied total intervals $\sum \tau_{f i}$ is shown. The parameters of the experiments are described above each figure. The black dots are the experimental results. The red solid line is a numerical simulation taking into account the momentum dispersion.

corrected simulations agree with the experiments quite well.

\section{MANIPULATE THE MOMENTUM STATES AS DESIGN}

The experiments and the numerical simulations above have shown the possibility and feasibility of the manipulation of a condensate's momentum states. We manage to design several two-pulse sequences to achieve high contrast momentum states such as $| \pm 2 \hbar k\rangle,| \pm 4 \hbar k\rangle$ and $| \pm 6 \hbar k\rangle$, which may be useful in atomic interferometry [19, 20]. For each state, we apply two totally different two-pulse sequences to show the flexibility of the method. The general method to achieve the target states is to find out the condition of the minimum of the square deviation $\Delta^{2}=\sum_{m=-\infty}^{+\infty}\left(P_{m}^{g}-P_{m}\right)^{2}$, where $P_{m}^{g}$ is the probability of $\left|2 m \hbar k_{L}\right\rangle$ in the goal state, and $P_{m}$ is that generated by the sequence. A second method, as the target is to obtain the highest population of some certain momentum state, consists in scanning the whole set of initial conditions and choose the one corresponding to the maximum value of the desired population. We apply the two methods above separately and obtain the same pulse sequences. As shown in Fig. [5, the experimental results (the black round dots) agree well with the expectations of the designs (the blue diamond dots), whether the pulses are in the Raman-Nath regime (see Fig. 囵(b)) or not (see others in Fig. (5). When the momentum dispersion is being considered, the expected momentum distributions (the red square dots) get closer to the experiments, where the figures only display the relative populations of the target states and omit the others for the figures being more clear.

It can be seen from Fig. 5 that the momentum width correction can improve the precision of the prediction
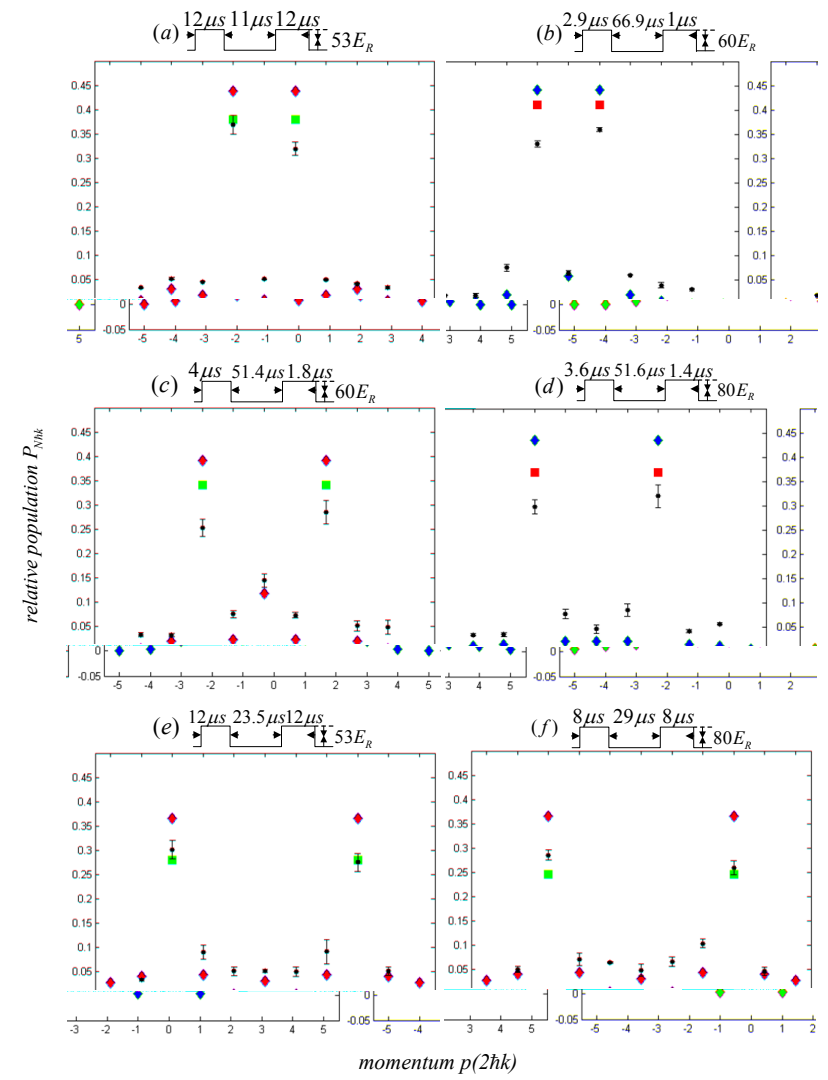

FIG. 5: Experimental realization of designed momentum states. The expected momentum state is $\pm 2 \hbar k_{L}((\mathrm{a})$ and (b)), $\pm 4 \hbar k_{L}((\mathrm{c})$ and $(\mathrm{d}))$ and $\pm 6 \hbar k_{L}((\mathrm{e})$ and (f)). The pulse sequences are shown above each figure. The black round dots are experimental results. The blue diamond dots are the expectations based on the design. The red square dots are the modified design with momentum width, which agree with the experiments better.

with our method. The average relative deviation between the experimental results and the expected values without including the momentum width is $25.03 \%$, while the deviation is decreased to $13.15 \%$ with the correction.

An asymmetry of the momenta can be observed in Fig. [5 and it may ascribe to the following factors. Besides the measurement error, there is an imperfection of the standing wave, brought forth by the unbalanced intensity of the laser beams. External field (such as the magnetic trap) fluctuations during the scattering process may also affect the momentum distribution.

\section{DISCUSSION AND CONCLUSION}

The band structure theory is a global method to deal with the standing wave scattering a condensate, while Bragg and Raman-Nath scattering are two special situations which can be analytically solved with their respective approximations. In the Bragg regime, the poten- 
tial height introduced by the standing wave is restrained below $4 E_{R}$ and that leads to the difficulty of generating higher order momentum states. In the Raman-Nath regime, the intensity of the standing wave is not limited so that higher order momentum states can be generated symmetrically [21, 22]. However the pulse duration has to be short enough to neglect the atomic motion, so the momentum states can not be predicted in this regime if the pulse duration is slightly longer. In our work, the scattering can be well explained and numerically analyzed within a much wider range of pulse intensity and duration. So it is natural that more momentum states can be generated.

In our article, we compared the scattering by one pulse and that by a train of pulses. Some valuable states, such as $| \pm 2 \hbar k\rangle,| \pm 4 \hbar k\rangle$ and $| \pm 6 \hbar k\rangle$ states with high contrast, can not be realized by the single pulse scattering, while they can be realized by a sequence of standing wave pulses. A sequence of lattice pulses is a more effective and flexible tool for momentum manipulation. It can generate much more useful momentum states than the ones demonstrated in our work. In the future, more parameters could be changed for the design to obtain better results.

Even though the numerical simulation is corrected to take into account the momentum dispersion, some deviations between the experiments and the simulation still exist. The inaccuracy of the lattice-depth calibration, which is $5 \%$ at least, is one of the reasons. The phase shift introduced by the magnetic trap is another one, while the influence is estimated to be within $0.03 \%$, which is below the experimental uncertainty. The heating and momenta exchange during the s-wave scattering may also lead to some differences, which need further study.

In conclusion, we developed a method for more flexible manipulation of the condensate's momentum states, where the momentum states can be controlled by standing wave pulses in a wider range of pulse intensity, duration or quantity. The experiments of a condensate being scattered by a sequence of standing wave pulses are demonstrated. A global theory, treating the standing wave as an optical lattice, is applied to explain the experiments. With this theory, we are able to design pulses sequences for realizing states such as $| \pm 2 \hbar k\rangle,| \pm 4 \hbar k\rangle$ and $| \pm 6 \hbar k\rangle$, and experimentally realize them, which may be applied in atomic interferometry to improve the measurement precision.

\section{ACKNOWLEDGEMENT}

We would like to thank Thibault Vogt for critical reading of our paper. This work is supported by the National Fundamental Research Program of China under Grant No. 2011CB921501, the National Natural Science Foundation of China under Grant No. 61027016, No.61078026, No.10874008 and No.10934010.
[1] Alexander D. Cronin et al., Rev. Mod. Phys. 81, 1051 (2009).

[2] Yoshio Torii et al., Phys. Rev. A 61, 041602 (2000).

[3] R. E. Sapiro et al., Phys. Rev. A 79, 043630 (2009).

[4] L. Deng et al., Phys. Rev. Lett. 83, 5407 (1999).

[5] M. Kozuma et al., Phys. Rev. Lett. 82, 871 (1999).

[6] Y. B. Ovchinnikov et al., Phys. Rev. Lett. 83, 284 (1999).

[7] C. Keller, et al., Appl. Phys. B-Laser and Optics, 69,303 (1999).

[8] S. Gupta et al., C. R. Acad. Sci. Paris 2, 479 (2001).

[9] D. E. Pritchard et al., Ann. Phys.-Berlin 10, 35 (2001).

[10] P. Meystre, Atom Optics(Springer-Verlag, New York, 2001)

[11] S. Wu et al., Phys. Rev. A 71, 043602 (2005).

[12] M. Edwards, et al., Phys. Rev. A 82, 63613 (2010).
[13] O. Morsch, et al., Rev. Mod. Phys. 78, 179 (2006).

[14] J. H. Denschlag et al., J. Phys. B-At. Mol. Opt. 35, 3095 (2002).

[15] F. Yang et al., Phys. Rev. A 78, 043611 (2008).

[16] X. J. Zhou et al., Phys. Rev. A 81, 013615 (2010).

[17] J. H. Huckans, et al. http://arxiv.org/abs/0901.1386 (2009).

[18] K. Li et al., Phys Rev Lett 101, 250401(2008).

[19] S. Beattie et al., Phys. Rev. A 80, 013618(2009).

[20] B. Rohwedder, Eur. Phys. J. D 13, 255 (2001).

[21] R. E. Sapiro, R. Zhang, and G. Raithel, New J. Phys. 11, 013013(2009).

[22] B. Gadway et al., Opt. Express 17, 19173 (2009). 